\title{
The formation of the quality parameters of the surface layer by ultrasonic
}

\author{
Vyacheslav Shuvaev ${ }^{1}$, and Nadezhda Krylova ${ }^{1, *}$ \\ ${ }^{1}$ Samara Technical University, 443100 Samara, Russia
}

\begin{abstract}
In work the questions of hardening of a surface layer of a detail by means of ultrasonic fluctuations are opened. The developed device for forming ultrasonic longitudinal-torsional oscillations is described, which makes it possible to substantially modify the properties of the surface layers of the parts.
\end{abstract}

It is known that the destruction of parts during operation, most often begins with the surface. Surface layers located on the interface between phases are subject to active action of the external environment, being the most strained. In this connection, there is a need to improve the physical and mechanical characteristics and geometry of the working surfaces of the parts. The state of the working surface of the part affects its operational properties such as wear resistance, fatigue strength, corrosion resistance, etc. The lack of stability in the quality of engineering products has a negative impact on the solution of socio-economic and scientific and technical problems, and therefore, issues of developing fundamentally new technologies, intensification of works in the field of quality engineering, ensuring the production of the highest quality products at a given production and the least labor costs.

One of the most effective and economical types of hardening is surface plastic deformation (PAP), which makes it possible to more fully realize the potential properties of structural materials in real details, especially in complex shape components with stress concentrators [1].

Ultimately, ultrasonic technologies have become increasingly widespread in machine building, the main features of which, determining their technological promise, are:

- high concentration of vibrational energy introduced into the zone of contacting the parts, which ensures local effects at significantly lower energy costs;

- low inertia of the process, which is the result of direct conversion of electrical energy into the energy of ultrasonic technological impact;

- the possibility of using oscillations that are excited in the process of ultrasonic vibrations, both for technological and diagnostic purposes.

The processes of re-deformation and destruction of the materials of the surface layers can be caused by various mechanisms acting at the microlevel, where the activation of one or another mechanism is determined by a combination of the values of the external factors acting on the material. The most significant effect on the strength of materials during

\footnotetext{
${ }^{*}$ Corresponding author: kryl.nad@gmail.com
} 
ultrasonic assembly is provided by voltages, frequency and amplitude of ultrasonic vibrations, temperature, and also the chemical activity of the working environments.

One of the effective methods to solve problems arising when the PPD is the imposition of ultrasonic vibration, making it possible to significantly modify the properties of the surface layers of parts by high energy impact harmonic oscillations of ultrasonic frequency, to control, within certain limits microgeometry formed surface, its hardness, the degree of work hardening as well as receive regular microrelief [2]. Under the influence of the tool (indenter) static force under constant pressure with a simultaneous superposition of the ultrasonic waves with a frequency of $18-24 \mathrm{kHz}$ is subjected to smoothing. The main advantages of ultrasonic PDP determined high performance and ease processing, as well as small constant pressure on the treated surface, the possibility of using universal machine tools.

The introduction of ultrasonic vibrations of high intensity into a metal in the solid state causes an increase in the density of structural imperfections (dislocations, vacancies), which leads to a change in the physico-mechanical properties of the metal being processed and affects the process of plastic deformation. At the interface between the solid phase and the solid phase, ultrasonic oscillations lead to a change in the state of the surface layer, reduce the forces of boundary friction when one body moves to another. Analysis of the research results shows that, regardless of the composition and initial mechanical properties of metals under ultrasound loading, the conditional yield stress decreases by a factor of 3.5-4 and the coefficient of friction by a factor of 2 to 4 . Ultrasonic vibrations reduce the static yield stress similar to heating, however, in order to achieve the same effect, ultrasound exposure requires significantly less energy than when heated. This difference is explained by the fact that ultrasonic energy is absorbed in those places of the crystal lattice that are carriers of the mechanism of plastic deformation (dislocation, grain boundaries, etc.), and is almost not absorbed in crystal-free zones of crystals [2].

In the process of ultrasonic surface plastic deformation, there is simultaneous static and dynamic loading, periodically repeated with a frequency of oscillations and propagating to a relatively small area of plastic deformation of the surface of the part. The ultrasonic treatment reduces the initial roughness of the surface of the part and forms residual compressive stresses, increases the microhardness of the surface and ensures its favorable distribution over the depth of the modified layer, contributes to the formation of an optimal microrelief for oil pockets, which ultimately provides an increase in wear resistance, cyclic strength, durability and reliability of machine parts [3].

There are known devices for ultrasonic surface treatment [4], the disadvantages of which are the complexity of the design, the need to use two vibration transducers to form a complex trajectory of the deforming element, in addition, there are no means to change the formed regular microrelief of the surface.

In pic. 1 shows an ultrasonic device developed by the authors for forming the microrelief of surfaces. The device comprises a concentrator 3 at the output end of which is placed a deforming element-ball 2, which acts on the surface to be treated 1 . To excite ultrasonic vibrations, a piezoceramic transducer 5 , clamped by means of a pin 7 and a nut 8 between the concentrator 3 and the counterweight 6 , is applied. The surface of the concentrator is provided with inclined grooves 4 , which allow exciting longitudinallytorsional vibrations of the deforming element. In addition, the deforming element is displaced relative to the axis of the concentrator, which leads to the formation of a complex trajectory of vibrating action on the surface of the workpiece.

The device works as follows.

The converter 5 excites longitudinal ultrasonic oscillations with a frequency of 18,000$23,000 \mathrm{~Hz}$ and an amplitude of up to $10-20 \mu \mathrm{m}$. The oscillation transducer 5 can be made, for example, on the basis of a packet piezoceramic transducer from piezoceramic washers 
of axial polarization of the type CTS-19. When a sine wave is applied from the voltage generator and the resonant frequency (not shown in the drawing) to the lining of the packet piezoceramic transducer, it changes its geometric dimensions by virtue of the reverse piezoelectric effect, excites mechanical oscillations of the concentrator 3 and, accordingly, of the deforming element 2 . Ultrasonic longitudinal oscillations, generated by the oscillation transducer 5, are transmitted to the concentrator 3 .

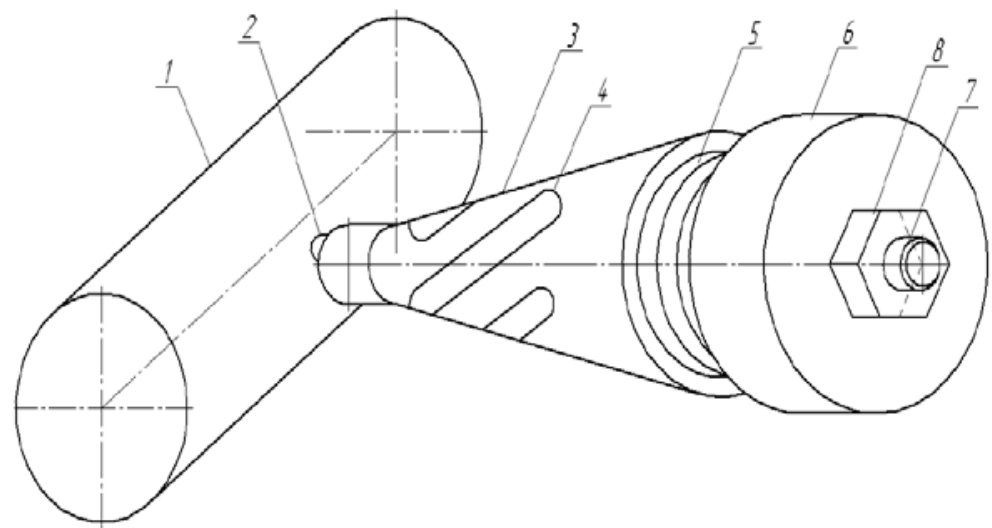

Fig. 1. Scheme of the device for ultrasonic surface treatment of parts.

It is possible to move the deforming element relative to the center of the output end of the concentrator perpendicular to the axial line of the cylindrical workpiece being processed. In Fig. 2a shows a variant with the displacement of the deforming element relative to the center of the output end of the concentrator perpendicular to the axial line of the cylindrical workpiece being processed.

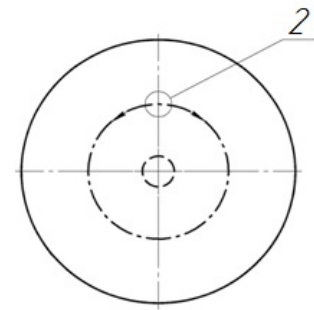

a

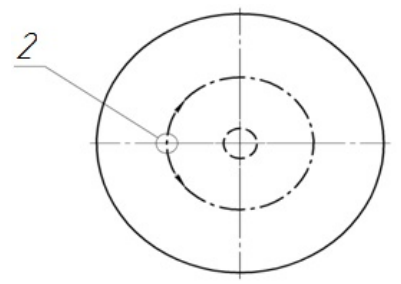

$\mathrm{b}$

Fig. 2. Displacement of the deforming element relative to the center of the output end of the concentrator: a - perpendicular to the axial line of the cylindrical workpiece being processed; $b$ parallel to the axial line of the cylindrical part being machined.

Similarly, the deforming element can be offset relative to the center of the output end of the concentrator parallel to the axial line of the cylindrical workpiece being processed. In Fig. $2 b$ shows a variant with the displacement of the deforming element relative to the center of the output end of the concentrator parallel to the axial line of the cylindrical workpiece being processed.

The displacement of the deforming element relative to the center of the output end of the concentrator perpendicularly or parallel to the axial line of the cylindrical part being processed makes it possible to form various types of regular surface microreliefs. The execution of inclined grooves on the outer surface of the concentrator leads to the formation of longitudinal-torsional oscillations, simplification of the design, and a reduction in the cost of the device for ultrasonic finishing of the outer cylindrical surfaces of the parts. 
In the process of ultrasonic vibratory actions on the surface of the workpiece, rapid deformations arise in it, leading to the excitation of elastic and plastic stress waves, and the forces arising in the process of ultrasonic treatment change in harmonic law with allowance for a constant component determined by the pressing force of the indenter to the workpiece.

The formation of complex vibrational fields in the process of ultrasonic treatment increases the efficiency of application and extends the possibilities of controlling ultrasonic treatment, and the displacement of the deforming element from the axis of the concentrator to different distances and at various angles relative to the axis of the workpiece significantly expands the types of regular surface microreliefs formed. The superposition of ultrasonic vibrations leads to a pulsed action of the indenter on the surface of the workpiece, the rotational and translational motion of the tool, and also the linear movement of the tool along the axis of the component, ensures a regular surface microrelief. The pattern resulting from the application of the oscillations varies depending on the following components: the frequency of the oscillations, the angle of displacement of the tip (relative to the center), the speed of the tool along the body of the part, the speed of rotation of the part itself.

Variable parameters of the processing process include the rotation speed of the part $n$ (rpm), the feed rate $\mathrm{s}(\mathrm{mm} / \mathrm{rev})$, the static force of pressing the deforming element against the machined surface $\mathrm{P}(\mathrm{H})$. Variable parameters of the ultrasonic oscillatory system are the operating frequency $\mathrm{f}=18-23 \mathrm{kHz}$, the amplitude of the ultrasonic vibrational influences $\mathrm{A}$ $=2-20 \mu \mathrm{m}$. The diameter of the deforming element - indentor can vary depending on the requirements for the surface parameters of the part and processing conditions $d=4-10 \mathrm{~mm}$.

The result of the addition of the transverse, longitudinal movement of the tool, as well as the longitudinally-torsional vibrations of the deforming element, is a complex vibrational effect on the surface to be treated.

The formation of complex vibrational fields in the process of ultrasonic treatment increases the efficiency of application and extends the possibilities of controlling ultrasonic treatment, and the displacement of the deforming element from the axis of the concentrator to different distances and at various angles relative to the axis of the workpiece significantly expands the types of regular surface microreliefs formed.

The technical result of the proposed development is to increase the efficiency and expand the technological capabilities of ultrasonic finish machining of the outer cylindrical surfaces of parts in order to improve their performance characteristics, improve the quality of the treated surface, increase the reliability and durability of its work, and shorten the running-in period. The simplicity of the design of this device allows the use of standard elements and applies it without additional costs for changing equipment and use universal lathes. The device can be placed on the lathe support, and the workpiece can be mounted in a chuck or centers.

The conducted laboratory tests of the developed ultrasonic device showed high efficiency of finishing-hardening processing of parts such as bodies of revolution. The introduction of ultrasonic vibrations into the zone of deformation led to an increase in the wear resistance of the surface layer by a factor of 1.5 to 2 as compared with conventional PPD methods, the area of the relative reference surface increases by $35-50 \%$ with the depth of the riveted layer up to $1 \mathrm{~mm}[5]$.

\section{References}

1. V.M. Smelyansky Mechanics of hardening of parts by surface plastic deformation. Moscow: Mechanical Engineering, (2002). - 300 p.

2. V.G. Shuvaev, V.A. Papshev, I.V Shuvaev. A tool for forming quality parameters of the surface layer of parts during ultrasonic finishing // STIN. (2012). № 10. P. 37-40. 
3. Patent of the Russian Federation No. 2393076. Method of ultrasonic finishing of external cylindrical surfaces / V.G. Shuvaev, V.A. Papshev, I.V. Shuvaev // 21.06.2010. Bul. No. 18.

4. A.V. Kirichek, D.L. Soloviev Technology and Regimes of Hardening by Static-Impulse Processing // Handbook. Engineering Journal. (2003). № 2. Pp. 17-19.

5. V.G. Shuvaev, N.A. Krylova Influence of ultrasonic treatment modes on the properties of cylindrical surfaces // International Symposium "Reliability and Quality", (2016). №2 P.185-186.

6. RF patent for utility model No. 143794. Device for ultrasonic finishing of external cylindrical surfaces of rotating parts / V.G. Shuvaev, M.S. Gorobets // 07/27/2014. Bul. No. 21. 